\title{
Melatonin Attenuates Chronic Cough Mediated by Oxidative Stress via Transient Receptor Potential Melastatin-2 in Guinea Pigs Exposed to Particulate Matter 2.5
}

\author{
Zhenjun JI $^{1}$, Zhen WANG ${ }^{1}$, Zhe CHEN ${ }^{2}$, Hao JIN ${ }^{1}$, Chen CHEN ${ }^{1}$, Senlin CHAI ${ }^{1}$, \\ Haining $\mathrm{LV}^{1}$, Ling YANG ${ }^{1}$, Yakun $\mathrm{HU}^{1}$, Rong DONG ${ }^{1}$, Kefang $\mathrm{LAI}^{2}$ \\ ${ }^{1}$ Medical School, Southeast University, Nanjing, China, ${ }^{2}$ State Key Laboratory of Respiratory \\ Disease, Guangzhou Institute of Respiratory Disease, The First Affiliated Hospital of Guangzhou \\ Medical University, Guangzhou, China
}

Received April 18, 2017

Accepted September 12, 2017

On-line January 5, 2018

\section{Summary}

The aim of this study was to investigate the effects of melatonin on oxidative stress, the expression of transient receptor potential melastatin-2 (TRPM2) in guinea pig brains, and the influence of melatonin on oxidative stress in lungs and airway inflammation induced by particulate matter 2.5 (PM2.5). A particle suspension $(0.1 \mathrm{~g} / \mathrm{ml})$ was nasally administered to the guinea pigs to prepare a PM2.5 exposure model. Cough frequency and cough incubation period were determined through RM6240B biological signal collection and disposal system. Oxidative stress markers, including malondialdehyde (MDA), total antioxidant capacity (T-AOC), total superoxide dismutase (T-SOD), and glutathione peroxidase (GSH-Px), in the medulla oblongata were examined through spectrophotometer. Reactive oxygen species (ROS) were detected in the hypoglossal nucleus, cuneate nucleus, Botzinger complex, dorsal vagal complex, and airway through dihydroethidium fluorescence. Hematoxylin-eosin (HE) staining and substance $\mathrm{P}$ expression via immunohistochemistry revealed the inflammatory levels in the airway. TRPM2 was observed in the medulla oblongata through immunofluorescence and Western blot. The ultrastructure of the blood-brain barrier and neuronal mitochondria was determined by using a transmission electron microscope. Our study suggests that melatonin treatment decreased PM2.5-induced oxidative stress level in the brains and lungs and relieved airway inflammation and chronic cough. TRPM2 might participate in oxidative stress in the cough center by regulating cough.

\section{Key words}

Melatonin • Oxidative stress • Cough sensitivity

\section{Corresponding authors}

K. Lai, State Key Laboratory of Respiratory Disease, The First Affiliated Hospital of Guangzhou Medical University, Guangzhou Institute of Respiratory Disease, 151 Yanjiang Rd., Guangzhou, China. E-mail: klai@163.com and R. Dong, Medical School of Southeast University, 87 Dingjiaqiao, Nanjing 210009, China. E-mail: dongrongshengli@163.com

\section{Introduction}

Ambient particulate matter 2.5 (PM2.5) is defined as airborne particulate matter with aerodynamic diameters less than $2.5 \mu \mathrm{m}$ (Chen et al. 2016). PM2.5 can transport directly into the alveoli which is mainly derived from oil and coal combustion and vehicle emissions; this pollutant is composed of elemental carbon, organic carbon, aluminum, copper, nickel, sulfates, nitrates, polycyclic aromatic hydrocarbons $(\mathrm{PAH})$ and other compounds (WHO 2013). Air pollutants, including PM2.5, are closely related to respiratory symptoms and diseases, such as chronic cough, chronic obstructive pulmonary diseases (COPD), asthma, and lung cancer (Falcon-Rodriguez et al. 2016, Zhang et al. 2015). Chronic cough is defined as cough lasting more than eight weeks without abnormal pulmonary function, and cough hypersensitivity is a key pathophysiological 
mechanism of chronic cough (Lai et al. 2013). However, the exact mechanism of cough hypersensitivity is unclear.

Cachon et al. (2014) found that human lung epithelial cells secreted more cytokines including IL-1 $\beta$, IL-6 and TNF- $\alpha$ after exposed to PM2.5. PM2.5 could enter the blood through the alveolus-capillary barrier and penetrate the blood-brain barrier (BBB) or migrate via the olfactory nerve pathway into the brain and cause neuroinflammation, oxidative stress, and neuronal damage (Bos et al. 2014). PM2.5 is also related to central nervous system (CNS) diseases, such as neurodegenerative diseases. Levesque et al. (2011) demonstrated that subchronic exposure to diesel engine exhaust causes neuroinflammation and thus increases the expression of $\alpha$-synuclein, an early marker of neurodegenerative diseases. Calderon-Garciduenas et al. $(2015 \mathrm{a}, \mathrm{b})$ found that neurodegenerative diseases in children are triggered by exposure to particulate matters and ozone, and environmental factors and gene factors promote Alzheimer's disease. Cough is a special kind of respiratory activity and the medulla oblongata receives sensory signals from the cough receptors in airways via vagus nerves and superior laryngeal nerves as the respiratory center. The respiratory center comprises the Botzinger complex, pons respiratory group, solitary nucleus group, and raphe nucleus group. These neurons in respiratory nuclei which participate in the integration of the afferent cough information are thought as central cough generator, and further regulate the motion of muscles in airways and generate cough (Chung and Pavord 2008). Signals from periphery are also subsequently transmitted into the superior center above the brainstem and it alters emotion and cognition brain regions. Further studies have not yet to clarify whether central nuclei generate pathological changes induced by PM2.5 and affect chronic cough development.

PM2.5 stimulates the release of endogenous and exogenous free radicals, including reactive oxygen species (ROS) in the CNS, as a result, the cause of oxidant/antioxidant systems imbalance (Fagundes et al. 2015, Liu et al. 2015). Direct PM2.5 exposure likely induces oxidative stress and neurotoxicity in the hippocampus and cerebellum (Fagundes et al. 2015). As a multifunctional non-selective cation channel with $\mathrm{N}$-domain, the transient potential receptor melastatin-2 (TRPM2) channel exhibits a pyrophosphatase activity, which can be activated by ROS and adenosine diphosphatase ribose (ADPR), and functions as a sensor for oxidative stress. TRPM2 is distributed mainly in different parts of the mammalian brain, including the hippocampus, cortex, thalamus, midbrain, and medulla oblongata; this channel is also abundant in neurons and microglia (Naziroglu 2011, Ru and Yao 2014). High TRPM2 expression mediates extracellular calcium entry and induces cell death. TRPM2 is also involved in many disorders, such as traumatic brain injury (Yuruker et al. 2015), cerebral ischemia (Akpinar et al. 2016), type 2 diabetes (Sozbir and Naziroglu 2016), cancers, inflammation, and neurodegenerative diseases (Naziroglu 2011). As such, TRPM2 is a potentially effective target of many diseases. However, studies have not yet to determine whether TRPM2 could mediate the generation of oxidative stress in the respiratory center and airway, and further induce airway inflammation and cough hypersensitivity.

Melatonin, secreted by the pineal gland, regulates the sleep, circadian rhythms and act as an effective antioxidant. In various pathological states, melatonin and its metabolites can function as endogenous free radical scavengers and broad-spectrum antioxidants to scavenge ROS. Melatonin also elicits a neuroprotective effect and protects the vascular endothelium by scavenging ROS and inhibiting pro-inflammatory cytokine release and $\mathrm{Ca}^{2+}$ overload (Akpinar et al. 2016, Kahya et al. 2017, Kaisar et al. 2015, Ma et al. 2015). Melatonin was hypothesized to alleviate chronic cough which serve as a potential therapeutic drug for chronic cough.

This study was aimed to investigate whether melatonin could alleviate chronic cough induced by PM2.5 through relieving oxidative stress and also determine whether TRPM2 participates in oxidative stress in the brain and influences airway oxidative stress and airway inflammation.

\section{Materials and Methods}

Animals

75 healthy male Hartley guinea pigs (350-500 g, Jiangnan Experimental Animal Center) were used in this study (Fig. 1). Experiments in this study were approved by the Animal Care and Use Committee of the Medical School of Southeast University. The animals were divided into normal saline control group, PM2.5 exposure group and PM2.5 exposure+melatonin treatment group. The acquisition of PM2.5 and suspension preparations, and animal model preparing were similar to our previous study (Lv et al. 2016). The PM2.5 exposure group 
received a $200 \mu \mathrm{l}$ suspension $(0.1 \mathrm{~g} / \mathrm{ml})$ each time, nasally instilled twice a day, for 28 continuous days, while the normal saline control group received equivalent saline. The PM2.5 exposure+melatonin treatment group received melatonin $(10 \mathrm{mg} / \mathrm{kg} /$ day $)$ via intraperitoneal injection for 8 days, while PM2.5 exposure+saline treatment group received equivalent saline.

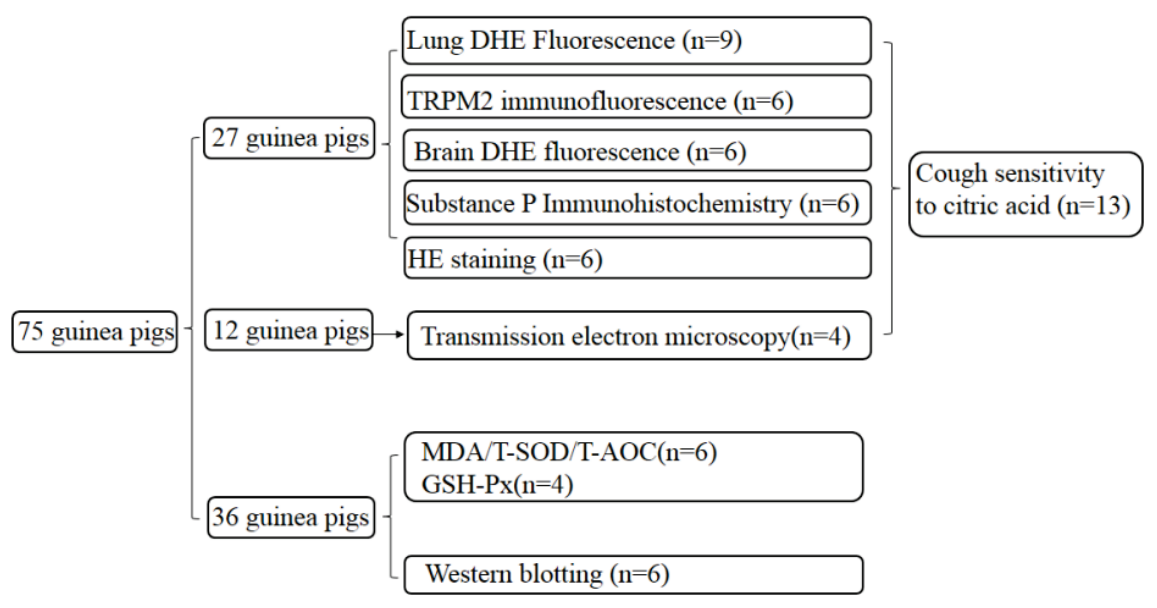

Fig. 1. The distribution of guinea pigs in various groups. All guinea pigs were randomly divided into 3 groups including normal saline control group, PM2.5 exposure group and PM2.5 exposure+melatonin treatment group. According to different handling methods, 27 guinea pigs were used for examination of DHE fluorescence, TRPM2 immunofluorescence, SP immunohistochemistry, and $\mathrm{HE}$ staining. 36 guinea pigs were used for Western blotting and examination of MDA/T-SOD/T-AOC/GSH-Px. Another 12 guinea pigs were used for observation by transmission electron microscopy.

\section{Cough sensitivity examination}

Cough frequency and cough incubation period were measured by RM6240B biological signal collection and disposal system (Chengdu, China). We prepared a $0.45 \mathrm{M}$ citric acid solution. Guinea pigs were placed in a cough recorder of sound energy, and the normal waveform was recorded for $10 \mathrm{~s}$ after the ultrasonic atomizer was opened and sprayed for $15 \mathrm{~s}$ into the recorder. We recorded cough frequency of guinea pigs within $10 \mathrm{~min}$ and time from the citric acid spray to the first cough of animals (cough incubation periods) to assess the cough reflex sensitivity in the airway of guinea pigs.

\section{Tissue preparation}

For immunofluorescence and dihydroethidium (DHE) fluorescence, guinea pigs were anesthetized by intraperitoneal injection of pentobarbital sodium (40 mg/kg), and then perfused and fixed with phosphate buffer containing $4 \%$ paraformaldehyde through the heart after chest opening. Then, the brain and lung tissues were placed in the fixed liquid for $8 \mathrm{~h}$, which were immersed in $30 \%$ sucrose solution for using after that.

\section{HE staining}

Lung tissues were embedded in paraffin wax after fixation in $4 \%$ paraformaldehyde for HE staining, and observed under light microscopy.

\section{Immunofluorescence}

The medulla oblongata was cut into continuous coronal slices $(30 \mu \mathrm{m}$ thickness per slice) from $2 \mathrm{~mm}$ below to $3 \mathrm{~mm}$ above the obex using a microtome (Leica, Germany). The slices were placed in sequence into phosphate buffered solution (PBS) containing $0.4 \%$ Triton X-100, PBS containing $10 \%$ goat serum (Abcam, UK), followed by incubation with a rabbit polyclonal anti-TRPM2 antibody (1:200, Abcam, UK) at $4{ }^{\circ} \mathrm{C}$ overnight, then rinsed in PBS for $5 \mathrm{~min}$. Subsequently, the sections were incubated with a goat anti-rabbit IgG/AlexaFluor 594 secondary antibody (1:400, Invitrogen, USA) at room temperature in the dark for $2 \mathrm{~h}$, followed by PBS rinsing ( $5 \mathrm{~min}$, three times), then dyed by DAPI for $1 \mathrm{~min}$ and rinsed in PBS for $5 \mathrm{~min}$. Finally, the slices were sealed with glycerol for observation by fluorescence microscope. The slices were observed by an Olympus fluorescence microscope (Olympus LSM-GB200, Japan) and cell number analysis was performed by Image-Pro Plus (Media Cybernetics, USA). Five random slices per animal were chosen for counting at a higher magnification $(200 \times)$. The average number of five slices represented the value per animal.

\section{Immunohistochemistry}

Lung tissue (size of $0.8 \times 0.6 \times 0.2 \mathrm{~cm}$ ) was cut into continuous coronal slices ( $45 \mu \mathrm{m}$ thickness per slice). Briefly, the slices were placed in deionized water containing $3 \% \mathrm{H}_{2} \mathrm{O}_{2}$, rinsed in PBS ( $5 \mathrm{~min}$, three times), incubated in rabbit serum (Abcam, UK) at room temperature for $4 \mathrm{~h}$, followed by incubation with goat anti-SP polyclonal antibody (1:200, Santa Cruz, USA) at 
$4{ }^{\circ} \mathrm{C}$ overnight, then rinsed in PBS for $5 \mathrm{~min}$. The slices were sequentially incubated with biotinylated rabbit antigoat IgG (Boster, China) for $2 \mathrm{~h}$, rinsed in PBS (5 min, three times), incubated with SABC (Boster, China) for $1 \mathrm{~h}$, and followed by PBS rinsing ( $5 \mathrm{~min}$, three times). The slices were stained with DAB (ZSGB-BIO, China) and dehydrated using graded ethanol and dimethylbenzene. The slices were sealed with a neutral resin under a microscope. Image-Pro Plus was used to calculate mean optical density (MOD) of SP in the lungs. The five random slices per animal were chosen for calculation and the mean data of five slices represented the value per animal.

\section{DHE fluorescence}

Tissues were prepared as discussed above. The slices were sequentially placed into PBS containing $0.4 \%$ Triton $\mathrm{X}-100$, followed by incubation with $2 \mu \mathrm{M}$ DHE working solution at $37^{\circ} \mathrm{C}$ for $40 \mathrm{~min}$ in the dark, and rinsing with PBS (5 min, three times). Finally, the slices were sealed with glycerol for observation by fluorescence microscope. Cell counting and fluorescence intensity were performed as described above.

\section{Measurement of peroxide and antioxidant enzymes}

The guinea pigs were decapitated directly and the medulla oblongata was rapidly stripped. Tissues were mechanically homogenized and mixed with saline using a ratio of weight $(\mathrm{g})$ : volume $(\mathrm{ml})=1: 9$ under an ice-water bath. The homogenates were centrifuged at 2,500 rpm for $10 \mathrm{~min}$. Furthermore, protein content of supernatant was measured by a 720 spectrophotometer. All procedures referred to the specifications of malondialdehyde (MDA)/total antioxidant capacity (T-AOC)/total superoxide dismutase (T-SOD)/glutathione peroxidase (GSH-Px) kits (Jiancheng, Nanjing, China).

\section{Western blot}

The medulla oblongata tissues of guinea pigs were homogenized in a lysis buffer (Beyotime, China) and were then centrifuged at $12,000 \mathrm{rpm}$ for $20 \mathrm{~min}$. Then, the samples were placed in $12 \%$ acrylamide denaturing gels (SDS-PAGE) and transferred to nitrocellulose membranes (Sigma, USA) after electrophoresis, followed by incubation for $1 \mathrm{~h}$ at room temperature with $5 \%$ non-fat dry milk in Tris Buffered Saline Tween (TBST). Then, the membranes were sequentially incubated with rabbit anti-TRPM2 (1:2,000, Abcam, USA) and HRP-linked goat anti-rabbit antibody
(1:5,000, Invitrogen, USA). Signals were captured by Microchemi chemiluminescent image analysis system (DNR Bio-imaging Systems, Jerusalem, Israel) after handling with the enhanced chemiluminescence method. Blots were quantified by Image-Pro Plus.

\section{Transmission electron microscopy}

Guinea pigs were anesthetized with $10 \%$ chloral hydrate (from $3 \mathrm{ml} / \mathrm{kg}$ to $4 \mathrm{ml} / \mathrm{kg}$, ip) and perfused with normal saline, followed by phosphate buffer containing $4 \%$ paraformaldehyde and $0.5 \%$ glutaraldehyde (Sigma, USA). Tissues in dorsal vagal complex (DVC) were diced and fixed in $2.5 \%$ chilled glutaraldehyde. A $1 \mathrm{~mm}^{3}$ tissue block was post-stained with uranyl acetate and lead citrate. Tissue sections were cut to 50-nm thicknesses and observed under a transmission electron microscope (JEOL, JEM-1010, Japan).

\section{Statistical analysis}

All data are analyzed by SPSS 19.0 and presented as the means \pm SEM. An independent-samples T-test was used to compare between two groups. Values of $p<0.05$ were considered statistically significant.

\section{Results}

\section{Cough sensitivity examination}

The cough frequency of the saline group, PM2.5 exposure group, and PM2.5 exposure+melatonin treatment groups were $8.46 \pm 1.02,25.92 \pm 2.74$, $18.77 \pm 1.77$, respectively, while the cough incubation periods were $68.46 \pm 8.38, \quad 35.69 \pm 6.17, \quad 57.77 \pm 7.26$. Compared with the saline group, the PM2.5 exposure group showed increased cough frequency $(p<0.001)$ and decreased cough incubation period $(p<0.01)$. After melatonin treatment, the PM2.5 exposure+melatonin treatment group showed decreased cough frequency $(p<0.05)$ and increased cough incubation period $(p<0.05)$ (Fig. 2). The results indicated that PM2.5 exposure increased cough sensitivity which was prevented by melatonin.

\section{Airway neurogenic inflammation and oxidative stress}

HE staining showed mucosa edema of the trachea and inflammatory cells infiltration, such as increased neutrophils, monocytes, lymphocytes and eosinophils, indicating exacerbated airway inflammation, which was relieved after melatonin treatment (Fig. 3A). 
A

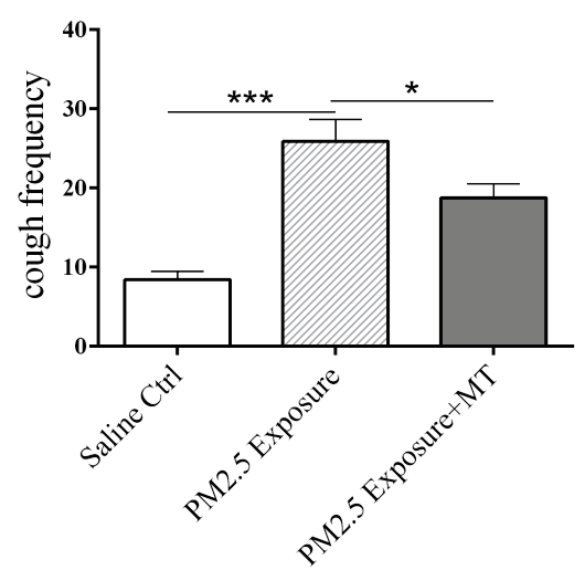

$\mathrm{B}$

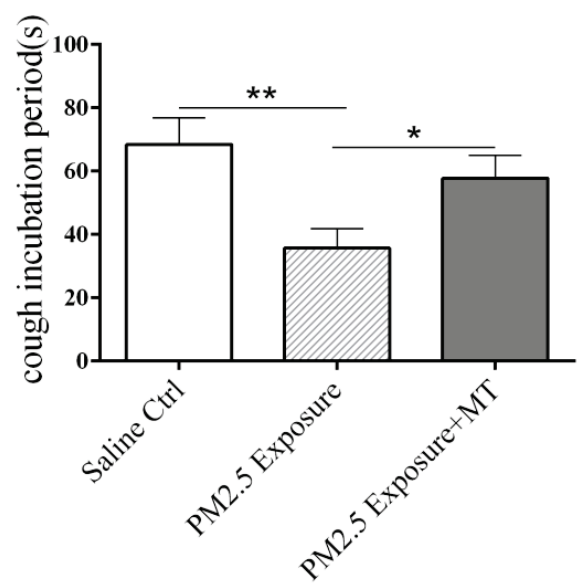

Fig. 2. Values for cough sensitivity examination. (A) Cough frequency showed significant differences between saline control group and PM2.5 exposure group, so was that between PM2.5 exposure group and PM2.5 exposure+melatonin group. (B) Cough incubation period of PM2.5 exposure group was less than saline control group, while PM2.5 exposure+melatonin group had prolonged period than PM2.5 exposure group. The values are presented as the means \pm SEM. ${ }^{*} p<0.05, * * p<0.01$, and $* * * p<0.001$, independent-samples T-test. $\mathrm{n}=13$ per group.

Immunoreactive substances of airway neurogenic inflammatory mediator substance $\mathrm{P}$ were dyed brown granules with cytoplasm staining and were mainly distributed around the airway. The MOD of substance $\mathrm{P}$ in the saline group, PM2.5 exposure group, and PM2.5 exposure+melatonin group were $0.19 \pm 0.03,0.42 \pm 0.05$, $0.21 \pm 0.04$, respectively. Compared with that in the saline group, the substance $\mathrm{P}$ expression increased after PM2.5 exposure $(p<0.01)$, while substance $\mathrm{P}$ expression decreased after melatonin treatment $(p<0.05)$ (Figs 3B and 3D). These results demonstrated that PM2.5 induced airway inflammation and melatonin had an antagonistic effect towards inflammation. DHE fluorescence of airway showed that fluorescence intensity of airway in PM2.5 exposure group is higher than that of the control group, which were decreased by melatonin $(p<0.05)$ (Figs $3 \mathrm{C}$ and 3E). The MOD of ROS in the saline group, PM2.5 exposure group, and PM2.5 exposure+melatonin group were $0.05 \pm 0.00,0.06 \pm 0.02, \quad 0.05 \pm 0.02$, respectively. These results indicate that PM2.5 exposure elevates the level of oxidative stress in the airway of guinea pigs, which were decreased by melatonin.

\section{Injury of $B B B$ and neurons}

The ultrastructure in the DVC observed by transmission electron microscope showed normal microvascular endothelium, astrocytes, and mitochondria structure with double membrane, and parallel arrangement of mitochondrial cristae in control group. The PM2.5 exposure group showed isolated microvascular endothelium, mild edema of astrocytes, and narrow vessels, which indicated injury of BBB. Besides, mitochondria were swollen and vacuolate, and were in disarray, with a decreased number of mitochondria and mitochondrial cristae. Essentially, damage to the BBB and mitochondria was relieved after intervention of melatonin (Fig. 4).

\section{Oxidative stress in medulla oblongata}

MDA represented the level of free radicals as lipid peroxides, while levels of T-AOC/T-SOD/GSH-Px represented the ability of scavenging free radical as antioxidant enzymes. Compared with the saline group, the PM2.5 exposure group showed increased levels of MDA representing the level of oxidation and decreased levels of T-AOC/GSH-Px representing antioxidant levels. However, decrease of T-SOD was not obvious significantly $(p<0.1)$. PM2.5 exposure+melatonin group showed obviously decreased level of MDA and increased levels of T-AOC/T-SOD/GSH-Px (Fig. 5A).

DHE marks the cytoplasm and nuclei of dead neurons producing ROS red (excitation $535 \mathrm{~nm}$, emission $610 \mathrm{~nm}$ ). Compared with the saline group, the PM2.5 exposure group showed an increased production of ROS in the hypoglossal nucleus, cuneate nucleus, Botzinger complex, and DVC $(p<0.05)$. Meanwhile, ROS production in PM2.5 exposure+melatonin group was decreased in relevant nuclei $(p<0.05)$. Our data showed that PM2.5 exposure induced oxidative stress in the medulla oblongata and melatonin treatment decreased the generation of ROS (Figs 5B and 5C). 


\section{TRPM2 expression in medulla oblongata}

The cytoplasms of immunoreactive neurons of TRPM2 were stained red under a fluorescence microscope. Compared with the saline group, the PM2.5 exposure group showed an increased expression of TRPM2 in the DVC and Botzinger complex $(p<0.01)$.
Melatonin treatment decreased the expression of TRPM2 $(p<0.05)$ (Figs 6A and 6B). Western blot showed that the protein level of TRPM2 in PM2.5 exposure group was higher than the saline and melatonin groups $(p<0.05)$ (Figs 6C and 6D).

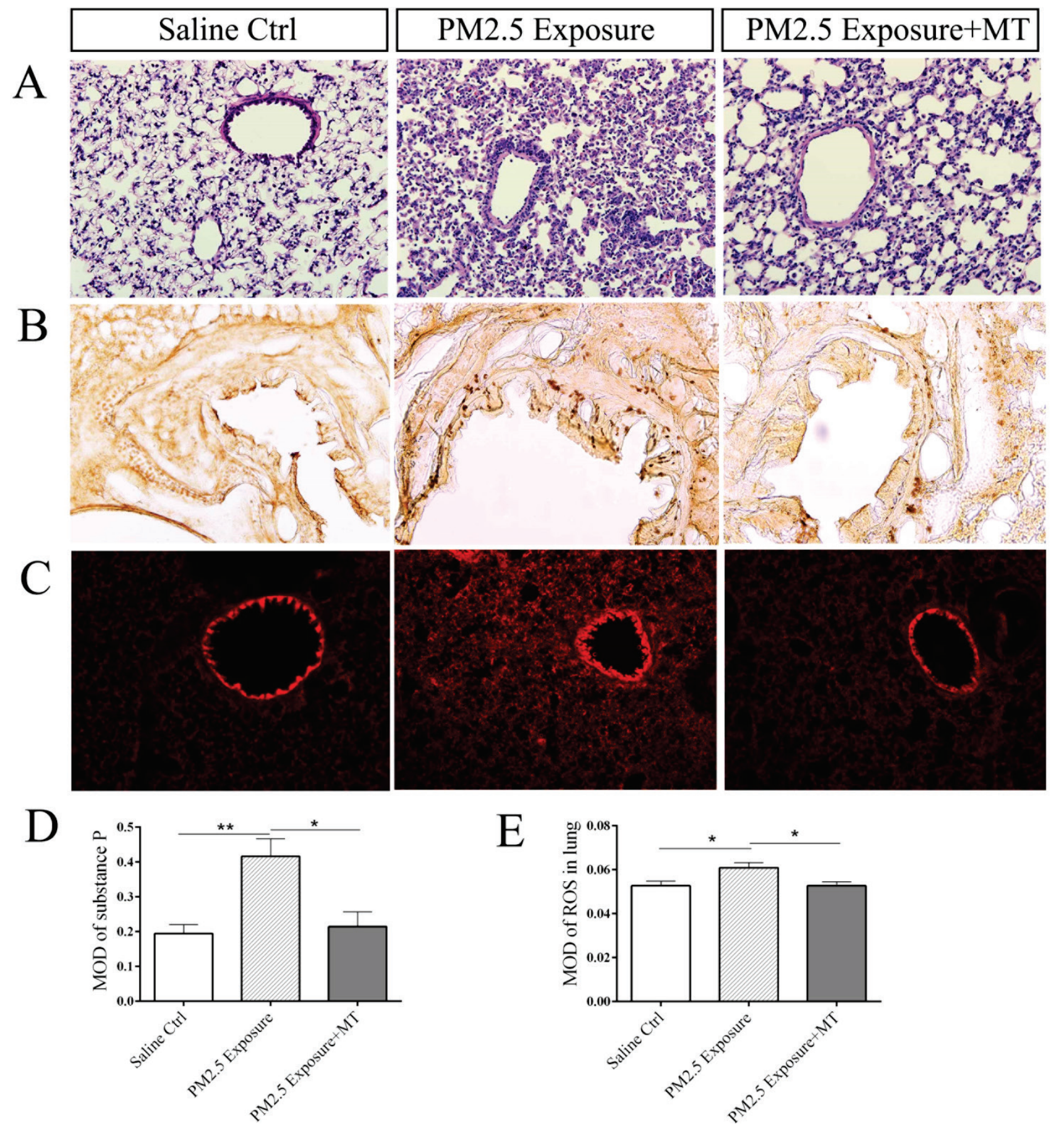

Fig. 3. Airway inflammation and oxidative stress in guinea pigs. (A) HE staining showing the pathological changes in lungs. (B) Representative images of substance P immunostaining. (C) Representative images of DHE fluorescence in airway. (D) The bar graph representing quantified results of substance $P$. Compared with that in the saline group, the substance $P$ expression increased after PM2.5 exposure $(p<0.01)$, while substance $\mathrm{P}$ expression decreased after melatonin treatment $(p<0.05)$. (E) The bar graph representing quantified results of lung ROS. Compared with that in the saline group, the MOD of ROS increased after PM2.5 exposure $(p<0.05)$, while the MOD values decreased after melatonin treatment $(p<0.05)$. The values are presented as the means \pm SEM. ${ }^{*} \mathrm{p}<0.05, * * \mathrm{p}<0.01$, independent-samples T-test. $\mathrm{n}=6-9$ per group. $\times 200$. 


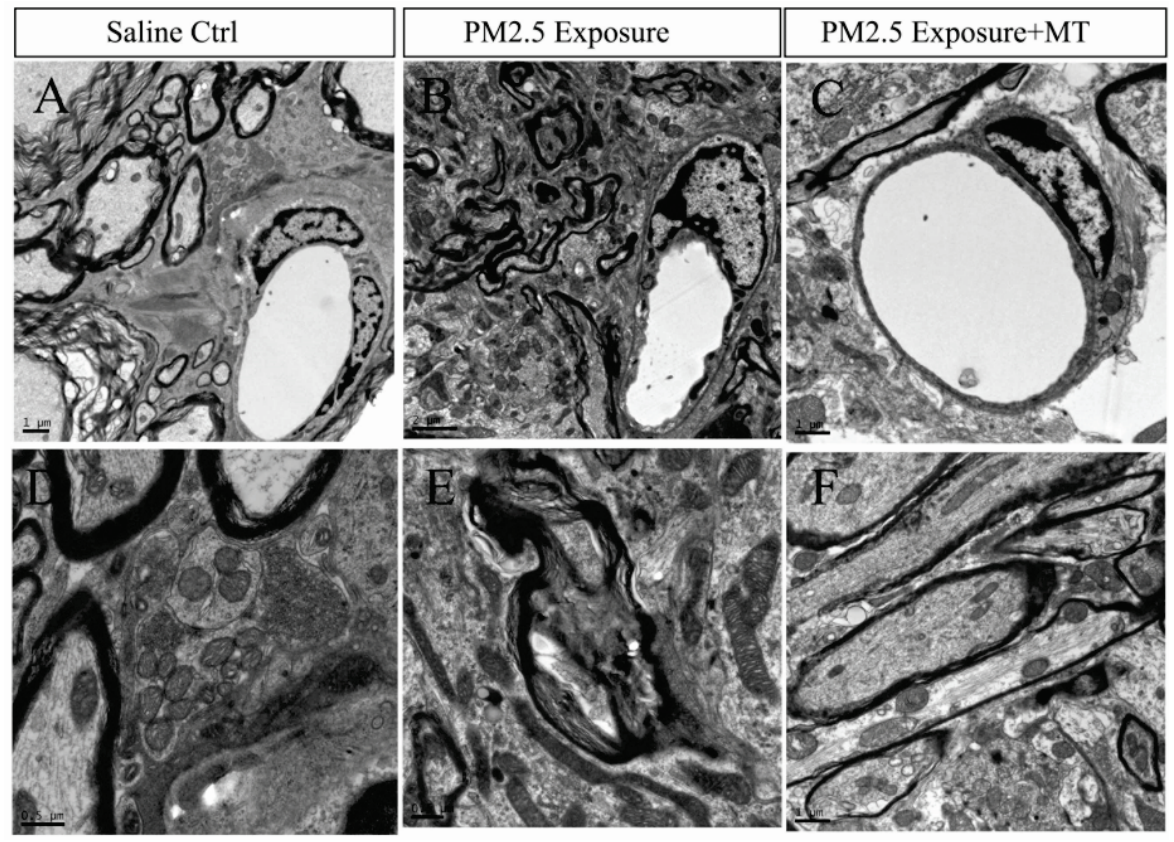

Fig. 4. Ultramicroscopic images of BBB and motochondria in dorsal vagal complex taken by transmission electron microscope. (A-C) Representative images of $\mathrm{BBB}$ in the saline control group, PM2.5 exposure group, and PM2.5 exposure group+melatonin treatment group. (D-F) Representative mitochondrial ultrastructure in three groups. Scale bars $=0.5 \mu \mathrm{m}$ in $\mathrm{D}$ and $\mathrm{E}$; scale bars $=1 \mu \mathrm{m} \mathrm{A}, \mathrm{C}$ and $\mathrm{F}$; scale bar $=2 \mu \mathrm{m}$ in $\mathrm{B}$.

\section{Discussion}

In our study, the particles were collected from five urban areas, including commercial areas, business areas, busy traffic intersections, industrial districts, and suburbs (Lv et al. 2016), and were mixed to represent the actual constituents of environmental PM2.5.

The increased cough sensitivity of guinea pigs after PM2.5 exposure showed that PM2.5 elicited chronic cough. We also found that PM2.5 exposure promoted the infiltration of inflammatory cells, including neutrophils, monocytes, lymphocytes, and eosinophils. Substance P expression was also increased which consequently induced the neurogenic inflammation in the airway; as a result, cause of airway inflammation which was consistent with the inflammatory phenomena of gastroesophageal reflux cough (GERC), cough variant cough (CVA) and chronic cough with definite etiology. Generally, guinea pigs after PM2.5 exposure showed cough hypersensitivity and the same pathological characteristics as chronic cough with definite etiology, which demonstrated that PM2.5 induced chronic cough. Airway neurogenic inflammation was induced by neuropeptides or neurotransmitters released by airway sensory nerve terminal, characterized by increased vascular permeability, plasma extravasation, and tissue edema. Substance P, Calcitonin Gene-Related Peptide (CGRP), and other substances stimulate vasodilation, plasma leakage, and mucus secretion, increase cough sensitivity, and trigger chronic cough. We further demonstrated that PM2.5 could increase oxidative stress levels, which could enhance the expression of inflammatory factors, such as interleukin (IL), via the NF- $\mathrm{KB}$ pathway and accumulate inflammatory cells. Airway inflammation and oxidative stress are two possible mechanisms exacerbating chronic cough, and ROS and inflammation are closely associated.

Cough is a special respiratory activity and respiratory defensive reflex. The respiratory center is located mostly in the medulla oblongata of brainstem, comprising the dorsal respiratory group with the solitary nucleus, and the ventral respiratory group containing the Botzinger complex. The stimuli of cough receptors including slowly and rapidly adapting stretch receptors (SAR and RAR) and C-fiber in the airway are transmitted to the solitary nucleus via the glossopharyngeal and vagus nerves to regulate the basic rhythm of inhalation. The DVC is composed of the dorsal nucleus of the vagus nerve (DMV), the nucleus of the solitary tract (NTS), and the area postrema. The DMV, hypoglossal nucleus, and nucleus ambiguus receive afferent fibers from the NTS (Kubin et al. 2006). The neuronal axons of the Botzinger complex projections are extensive in various regions of the medulla oblongata and spinal cord associated with breathing, and these axons inhibit the discharge of inhalation neurons during the expiratory phase to participate in maintaining the expiratory phase. Therefore, the mutual association between the central nervous system and airway has the neuroanatomical foundation. Afferent stimuli in the airway can reach the 
cough center in the brainstem including the DVC and the ventral Botzinger complex in the medulla oblongata through the sensory afferent nerve. The cough center also regulates respiratory and throat muscles after signals are integrated and further generates cough (Mazzone and Undem 2017).
A
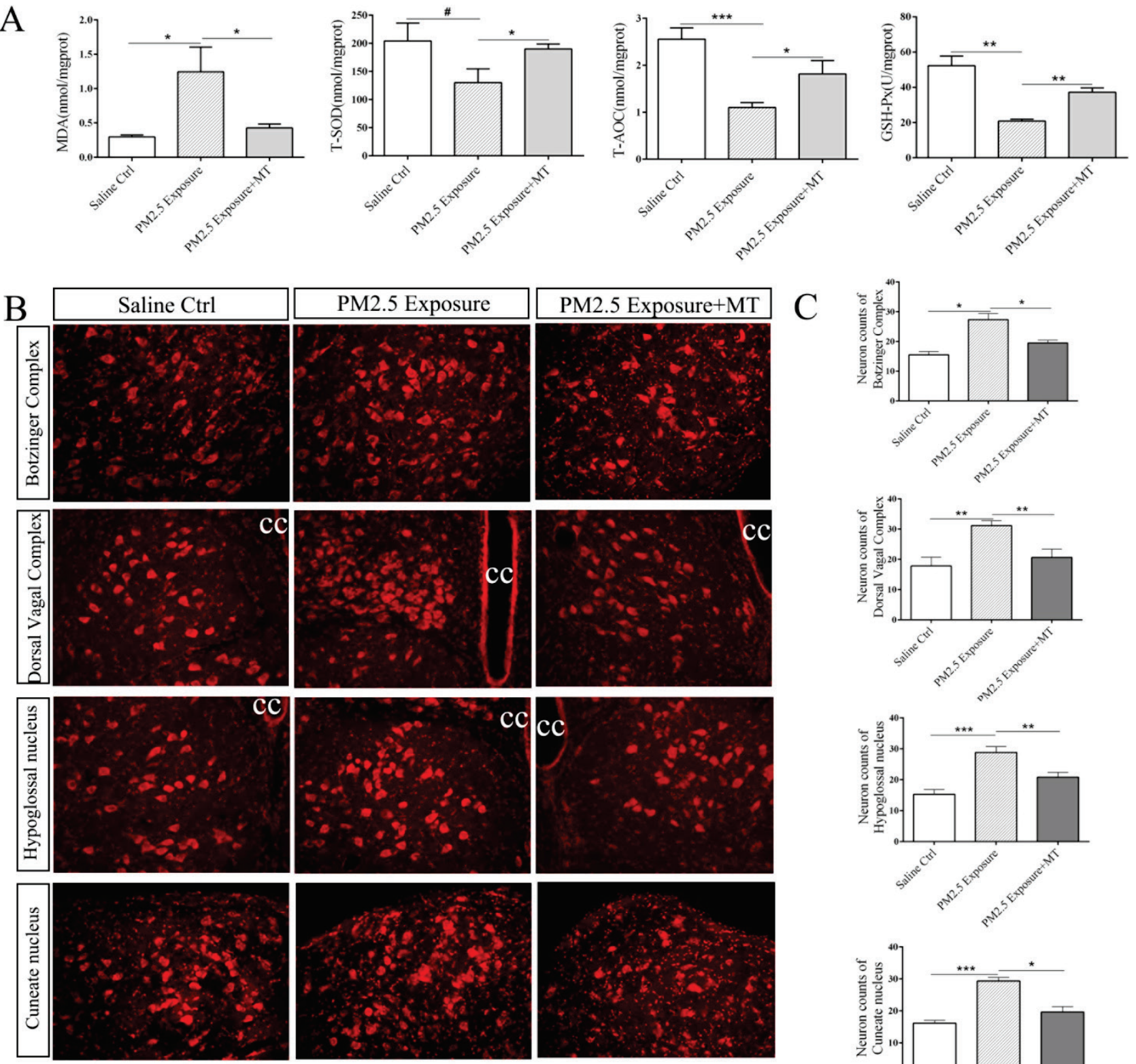
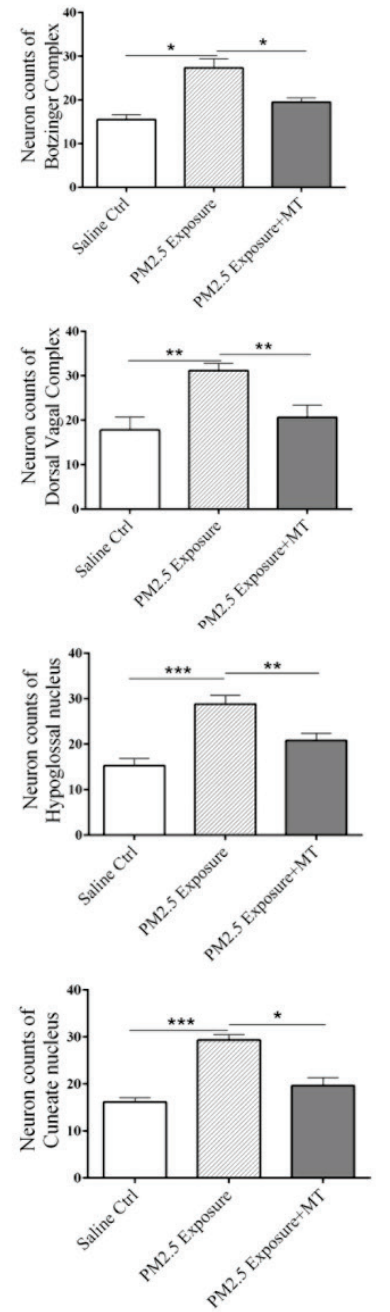

Fig. 5. Levels of oxidative stress of medulla oblongata. (A) Levels of MDA/T-SOD/T-AOC and GSH-Px in medulla oblongata of saline control group, PM2.5 exposure group and PM2.5 exposure group+melatonin treatment group. Compared with the saline group, the PM2.5 exposure group showed increased levels of MDA representing the level of oxidation $(p<0.05)$ and decreased levels of T-AOC $(p<0.001)$ and GSH-Px $(p<0.01)$ representing antioxidant levels. The decrease of T-SOD was not obvious significantly $(p<0.1)$. PM2.5 exposure+melatonin group showed obviously decreased level of MDA $(p<0.05)$ and increased levels of T-AOC/T-SOD $(p<0.05)$ and GSH-Px $(p<0.01)$. (B) Representative images of ROS stained by DHE fluorescence in hypoglossal nucleus, cuneate nucleus, Botzinger complex and dorsal vagal complex. (C) The bar graph representing quantified results of brain ROS respectively. Compared with the saline group, the PM2.5 exposure group showed an increased production of ROS in the hypoglossal nucleus $(p<0.001)$, cuneate nucleus $(p<0.001)$, Botzinger complex $(p<0.05)$, and DVC $(p<0.01)$. Meanwhile, ROS production in PM2.5 exposure+melatonin group was decreased in Botzinger complex $(p<0.05)$, cuneate nucleus $(p<0.05)$, hypoglossal nucleus $(p<0.01)$ and DVC $(p<0.01)$. The values are presented as the means \pm SEM. ${ }^{*} p<0.05, * * p<0.01, * * * p<0.001$, and ${ }^{\#} p>0.05$, independent-samples T-test. $n=4-6$ per group. cc - central canal. $\times 200$. 
A
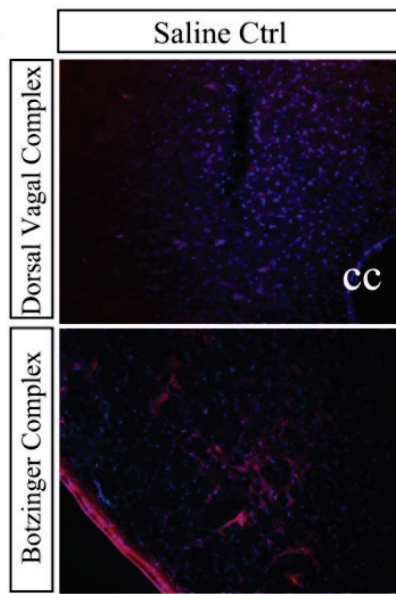
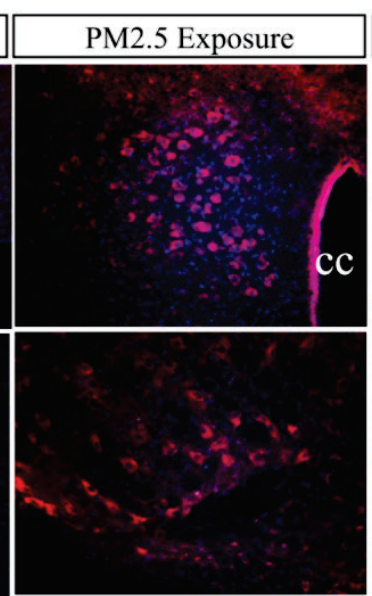

PM2.5 Exposure+MT
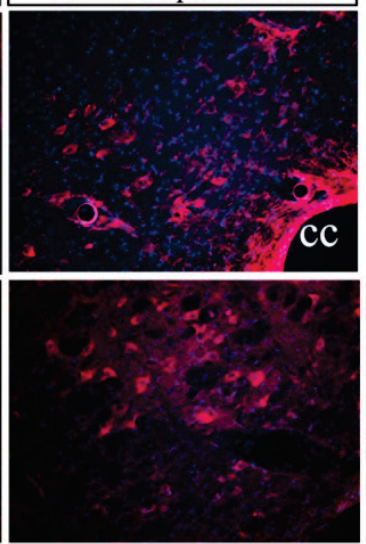

$\mathrm{C}$

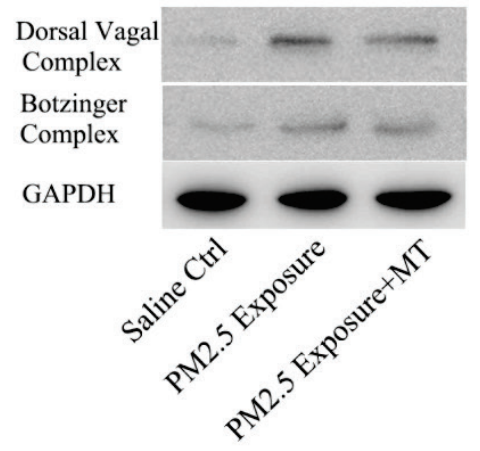

B
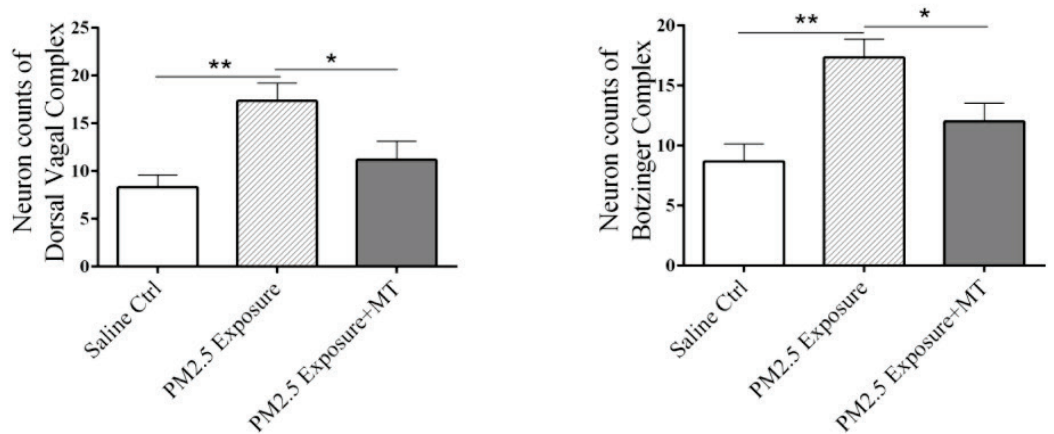

$\mathrm{D}$
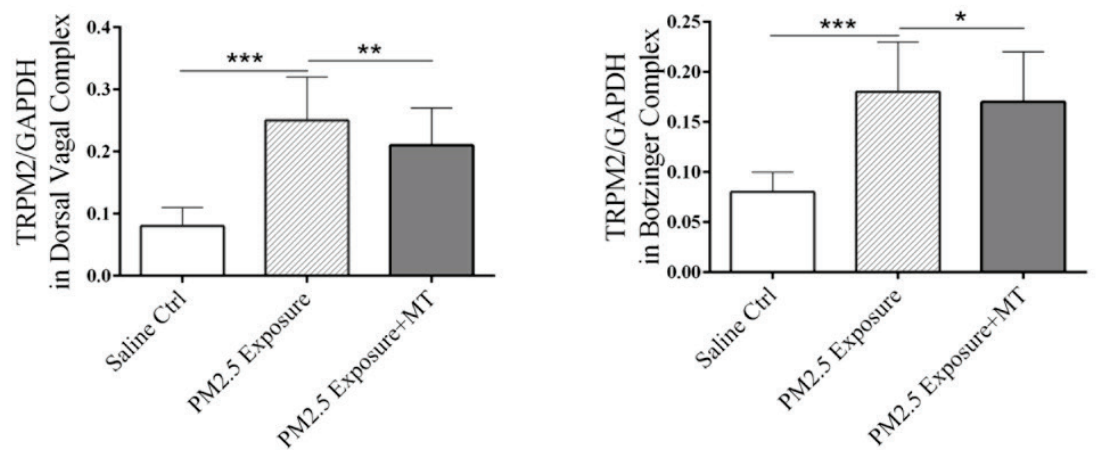

Fig. 6. Levels of TRPM2 in medulla oblongata of saline control group, PM2.5 exposure group and PM2.5 exposure group+melatonin treatment group. (A) Representative images of TRPM2 expression (red) in the Botzinger complex and dorsal vagal complex. Nuclei were counterstained with DAPI (blue). (B) The bar graphs representing quantified results of cell counting. Compared with the saline group, the PM2.5 exposure group showed an increased expression of TRPM2 in the DVC and Botzinger complex $(p<0.01)$. Melatonin treatment decreased the expression of TRPM2 ( $p<0.05)$ (Figs 5A and 5B). (C) Western blot showing protein level of TRPM2 in medulla oblongata. (D) The bar graphs representing quantified results of TRPM2. The protein level of TRPM2 in PM2.5 exposure group was higher than the saline group $(p<0.001)$ and PM2.5 exposure+melatonin group showed decreased protein level in the DVC $(p<0.01)$ and Botzinger complex $(p<0.05)$. The values are presented as the means \pm SEM. $* p<0.05, * * p<0.01$, and $* * * p<0.001$, independent-samples T-test. $\mathrm{n}=6 . \mathrm{cc}-$ central canal. $\times 200$.

BBB consists of vascular endothelial cell, tight junction between endothelium, astrocyte, pericyte and basement membrane. In the ultrastructure observed with a transmission electron microscope, isolated microvascular endothelium, mild edema of astrocytes, and narrow vessels were present, and this observation indicated that PM2.5 could damage the BBB. Liu et al. (2015) found that PM2.5 disrupts the tight junction of endothelial cells, increases penetrability, and enhances monocyte migration ability and demonstrated that PM2.5 can reach the CNS through the BBB; the neurotoxicity of PM2.5 is also mediated by glutamate (Liu et al. 2015). Swollen and vacuolate mitochondria were also observed in the ultrastructure, and the number of the mitochondria and mitochondrial cristae was decreased. These findings suggested that PM2.5 induced mitochondrial injury, which could trigger imbalance in calcium homeostasis and energy metabolism in cells, and neuronal injury or 
apoptosis. The leakage of the mitochondrial electron transport chain is also a vital source of intracellular ROS, leading to oxidant/antioxidant imbalance (RedzaDutordoir and Averill-Bates 2016). We also found that the MDA level was increased, whereas T-SOD, GSH-Px, and T-AOC levels were decreased after PM2.5 exposure. DHE fluorescence demonstrated that ROS production was increased in DVC, Botzinger complex, hypoglossal nucleus, and cuneate nucleus. These observations showed that the medulla oblongata was in a state of high oxidative stress after PM2.5 exposure. It was reported that cerebral ischemia, epilepsy, and trauma result in the generation of oxidative stress (Halliwell 2006), which was related to BBB endothelial damage and was relieved by antioxidants (Kaisar et al. 2015). Oxidative stress is also relevant to neurodegenerative diseases. Children exposed to air pollutants, including PM2.5, in Mexico City Metropolitan Area manifest signs and symptoms of early oxidative stress, inflammation, innate and adaptive immunity-related genes, and BBB disruption, resulting in the early neurodegenerative changes in children (Calderon-Garciduenas et al. 2015b). Hence, during the process of air pollutants inducing chronic cough, the activity of the cough reflex is strengthened, and oxidative stress in the center is enhanced as the generation of airway inflammation. Further, the cough center is remodeled, and chronic cough generated.

TRPM2 is a cation channel that exhibits oxidative stress sensitivity and mediates oxidative stressinduced cell death via $\mathrm{Ca}^{2+}$ overload ( $\mathrm{Ru}$ and Yao 2014). Our immunofluorescence results revealed that PM2.5 exposure increased the TRPM2 expression in respiratory nuclei, Botzinger complex, and DVC, whereas oxidative stress levels were increased. These findings were also confirmed through Western blot. The TRPM2 channel consists of six transmembrane segments with a poreforming loop between segments 5 and 6 . The N-terminal of TRPM2 contains an IQ-like calmodulin-binding motif and the C-terminal comprises a Nudix-like domain, which can be bound by ADPR. Calcium overload after TRPM2 activation in microglia and astrocytes can lead to mitochondrial dysfunction, and calcium overload is also related to synaptic plasticity change and dementia (Wang J. et al. 2016). TRPM2-mediated $\mathrm{Ca}^{2+}$ influx activated by oxidative stress inhibits autophagy (Wang Q. et al. 2016).

We utilized antioxidant melatonin to elucidate the role of ROS and TRPM2 in the guinea pig model of PM2.5 exposure. Melatonin treatment decreased the oxidative stress level in the medulla oblongata, the TRPM2 expression in the Botzinger complex and DVC. These findings indicated that ROS might be involved in TRPM2 activation. Yürüker et al. (2015) also found that melatonin alleviates oxidative stress and apoptosis by inhibiting $\mathrm{Ca}^{2+}$ and TRPM2 channels in the hippocampus of rats with traumatic brain injury; this observation suggests that melatonin exhibits neuroprotective activity (Yürüker et al. 2015). The GSH-Px levels changed most obviously possibly because melatonin maintains the glutathione balance by stimulating the generation of glutathione peroxidase, glutathione reductase, and glucose-6-phosphate dehydrogenase (Reiter et al. 2000). Hence, melatonin could upregulate the levels of T-SOD/GSH-Px/glutathione reductase by eliciting an indirect antioxidant effect and by directly scavenging free radicals and reducing MDA production. Kahya et al. (2017) demonstrated that melatonin and selenium can prevent the apoptosis of neurons in the hippocampus and dorsal root ganglion of diabetic rats by reducing ROS and calcium influx associated with TRPM2 and TRPV1 (Kahya et al. 2017). Melatonin can also function at the genetic level to prevent DNA degradation and activate DNA repair enzyme. Melatonin binding sites are also found in cell nuclei. Melatonin metabolites, namely, $N$-1-acetyl- $N$-2-formyl-5-methoxykynuramine and $N$-1-acetyl-5-methoxykynuramine, induce a similar antioxidant effect to melatonin. Transmission electron microscopy revealed that melatonin relieved the injuries of BBB and mitochondria in the DVC. The melatonin transporter located on the mitochondrial outer membrane and melatonin can be secreted by mitochondria and induce an agglomeration effect. The concentration of melatonin in the mitochondria is higher than that in other organelles. Melatonin can inhibit the mitochondrial permeability transition pore (MPTP) in the mitochondrial membrane and activate uncoupling proteins (UCPs). The inhibition of MPTP can maintain the mitochondrial membrane potential $(\Delta \Psi)$, which can be reduced by UCPs; thus, electron transfer can be accelerated, the efficiency of the mitochondrial electron transport chain can be improved, and electron leakage and ROS production can be reduced. Moreover, melatonin can prevent neuronal apoptosis by decreasing the levels of pro-apoptotic factors and by activating JAK2/STAT3 and BCL2 pathways (Ganie et al. 2016, Tan et al. 2016). The antioxidant edaravone can also alleviate brain injuries of acute CO poisoning rats ( $\mathrm{Li}$ et al. 2016). In cerebral ischemia rat models, dexmedetomidine generates 
a neuroprotective effect by reducing oxidative stress and inhibiting $\mathrm{Ca}^{2+}$ entry and apoptosis (Akpinar et al. 2016). Our experiment showed a decrease in cough sensitivity and reduced substance $\mathrm{P}$ expression and inflammation and oxidative stress levels in the airway after melatonin treatment. Therefore, melatonin could alleviate chronic cough induced by PM2.5 to some extent. Melatonin also shows an efficient anti-inflammation ability in airway hyper-reactivity (Chen et al. 2011), acute lung injury (Zhang et al. 2016), and neurogenic pulmonary edema (Chen et al. 2015) in rat or mouse models.

Our study demonstrated airway-generated inflammation, oxidative stress, and cough hypersensitivity in guinea pigs exposed to PM2.5. The cough center also showed high levels of oxidative stress and TRPM2, and injury of $\mathrm{BBB}$ and mitochondrial, but these conditions were alleviated by melatonin treatment. Therefore, TRPM2 might be involved in oxidative stress in the brain and regulation of peripheral inflammation by increasing calcium influx and neuronal apoptosis.

In conclusion, our findings mainly suggested that melatonin treatment could relieve cough by decreasing the expression of TRPM 2 in the cough center, alleviating oxidative stress in the brain or airway, and relieving airway inflammation. However, the exact role of TRPM2 in the brain region that regulates airway oxidative stress and inflammation should be further investigated on the basis of antagonist intervention or TRPM2 knockout mice.

\section{Conflict of Interest}

There is no conflict of interest.

\section{Acknowledgements}

This work was supported by Grants 2016 OP015 from Open Project of State Key Laboratory of Respiratory Disease and Grants 81570092 and 81770098 from the National Natural Science Foundation of China. Authors would like to thank Xinjian Zhu for his assistance of experiment.

\section{References}

AKPINAR H, NAZIROGLU M, OVEY IS, CIG B, AKPINAR O: The neuroprotective action of dexmedetomidine on apoptosis, calcium entry and oxidative stress in cerebral ischemia-induced rats: contribution of TRPM2 and TRPV1 channels. Sci Rep 6: 37196, 2016.

BOS I, DE BOEVER P, INT PANIS L, MEEUSEN R: Physical activity, air pollution and the brain. Sports Med 44: 1505-1518, 2014.

CACHON BF, FIRMIN S, VERDIN A, AYI-FANOU L, BILLET S, CAZIER F, MARTIN PJ, AISSI F, COURCOT D, SANNI A, SHIRALI P: Proinflammatory effects and oxidative stress within human bronchial epithelial cells exposed to atmospheric particulate matter $(\operatorname{PM}(2.5)$ and $\operatorname{PM}(>2.5))$ collected from Cotonou, Benin. Environ Pollut 185: 340-351, 2014.

CALDERON-GARCIDUENAS L, MORA-TISCARENO A, MELO-SANCHEZ G, RODRIGUEZ-DIAZ J, TORRESJARDON R, STYNER M, MUKHERJEE PS, LIN W, JEWELLS V: A critical proton MR spectroscopy marker of Alzheimer's disease early neurodegenerative change: low hippocampal NAA/Cr ratio impacts APOE epsilon4 Mexico City children and their parents. J Alzheimers Dis 48: 1065-1075, 2015a.

CALDERON-GARCIDUENAS L, VOJDANI A, BLAUROCK-BUSCH E, BUSCH Y, FRIEDLE A, FRANCO-LIRA M, SARATHI-MUKHERJEE P, MARTINEZ-AGUIRRE X, PARK SB, TORRES-JARDON R, D'ANGIULLI

A: Air pollution and children: neural and tight junction antibodies and combustion metals, the role of barrier breakdown and brain immunity in neurodegeneration. J Alzheimers Dis 43: 1039-1058, 2015 b.

CHEN CF, WANG D, REITER RJ, YEH DY: Oral melatonin attenuates lung inflammation and airway hyperreactivity induced by inhalation of aerosolized pancreatic fluid in rats. J Pineal Res 50: 46-53, 2011.

CHEN JY, QIAN C, DUAN HY, CAO SL, YU XB, LI JR, GU C, YAN F, WANG L, CHEN G: Melatonin attenuates neurogenic pulmonary edema via the regulation of inflammation and apoptosis after subarachnoid hemorrhage in rats. J Pineal Res 59: 469-477, 2015.

CHEN R, HU B, LIU Y, XU J, YANG G, XU D, CHEN C: Beyond PM2.5: the role of ultrafine particles on adverse health effects of air pollution. Biochim Biophys Acta 1860: 2844-2855, 2016.

CHUNG KF, PAVORD ID: Prevalence, pathogenesis, and causes of chronic cough. Lancet 371: 1364-1374, 2008. 
FAGUNDES LS, FLECK A DA S, ZANCHI AC, SALDIVA PH, RHODEN CR: Direct contact with particulate matter increases oxidative stress in different brain structures. Inhal Toxicol 27: 462-467, 2015.

FALCON-RODRIGUEZ CI, OSORNIO-VARGAS AR, SADA-OVALLE I, SEGURA-MEDINA P: Aeroparticles, composition, and lung diseases. Front Immunol 7: 3, 2016.

GANIE SA, DAR TA, BHAT AH, DAR KB, ANEES S, ZARGAR MA, MASOOD A: Melatonin: a potential antioxidant therapeutic agent for mitochondrial dysfunctions and related disorders. Rejuvenation Res 19: 21-40, 2016.

HALLIWELL B: Oxidative stress and neurodegeneration: where are we now? J Neurochem 97: 1634-1658, 2006.

KAHYA MC, NAZIROGLU M, OVEY IS: Modulation of diabetes-induced oxidative stress, apoptosis, and Ca2+ entry through TRPM2 and TRPV1 channels in dorsal root ganglion and hippocampus of diabetic rats by melatonin and selenium. Mol Neurobiol 54: 2345-2360, 2017.

KAISAR MA, PRASAD S, CUCULLO L: Protecting the BBB endothelium against cigarette smoke-induced oxidative stress using popular antioxidants: are they really beneficial? Brain Res 1627: 90-100, 2015.

KUBIN L, ALHEID GF, ZUPERKU EJ, MCCRIMMON DR: Central pathways of pulmonary and lower airway vagal afferents. J Appl Physiol (1985) 101: 618-627, 2006.

LAI KF, CHEN RC, ZHONG NS: Air pollution and chronic cough in China response. Chest 144: 363-364, 2013.

LEVESQUE S, SURACE MJ, MCDONALD J, BLOCK ML: Air pollution \& the brain: Subchronic diesel exhaust exposure causes neuroinflammation and elevates early markers of neurodegenerative disease. J Neuroinflammation 8: 105, 2011.

LI Q, BI MJ, BI WK, KANG H, YAN LJ, GUO YL: Edaravone attenuates brain damage in rats after acute CO poisoning through inhibiting apoptosis and oxidative stress. Environ Toxicol 31: 372-379, 2016.

LIU F, HUANG Y, ZHANG F, CHEN Q, WU B, RUI W, ZHENG JC, DING W: Macrophages treated with particulate matter PM2.5 induce selective neurotoxicity through glutaminase-mediated glutamate generation. J Neurochem 134: 315-326, 2015.

LV H, YUE J, CHEN Z, CHAI S, CAO X, ZHAN J, JI Z, ZHANG H, DONG R, LAI K: Effect of transient receptor potential vanilloid-1 on cough hypersensitivity induced by particulate matter 2.5. Life Sci 151: 157-166, 2016.

MA P, LIU X, WU J, YAN B, ZHANG Y, LU Y, WU Y, LIU C, GUO J, NANBERG E, BORNEHAG CG, YANG X: Cognitive deficits and anxiety induced by diisononyl phthalate in mice and the neuroprotective effects of melatonin. Sci Rep 5: 14676, 2015.

MAZZONE SB, UNDEM BJ: Vagal afferent innervation of the airways in health and disease. Physiol Rev 96: 975-1024, 2017.

NAZIROGLU M: TRPM2 cation channels, oxidative stress and neurological diseases: where are we now? Neurochem Res 36: 355-366, 2011.

REDZA-DUTORDOIR M, AVERILL-BATES DA: Activation of apoptosis signalling pathways by reactive oxygen species. Biochim Biophys Acta 1863: 2977-2992, 2016.

REITER RJ, TAN DX, OSUNA C, GITTO E: Actions of melatonin in the reduction of oxidative stress. $J$ Biomed Sci 7 : 444-458, 2000.

RU XC, YAO XQ: TRPM2: a multifunctional ion channel for oxidative stress sensing. Acta Physiol Sinica 66: 7-15, 2014.

SOZBIR E, NAZIROGLU M: Diabetes enhances oxidative stress-induced TRPM2 channel activity and its control by $\mathrm{N}$-acetylcysteine in rat dorsal root ganglion and brain. Metab Brain Dis 31: 385-393, 2016.

TAN DX, MANCHESTER LC, QIN L, REITER RJ: Melatonin: a mitochondrial targeting molecule involving mitochondrial protection and dynamics. Int J Mol Sci 17: 2124, 2016.

WANG J, JACKSON MF, XIE YF: Glia and TRPM2 channels in plasticity of central nervous system and Alzheimer's diseases. Neural Plast 2016: 1680905, 2016.

WANG Q, GUO W, HAO B, SHI X, LU Y, WONG CWM, MA VWS, YIP TTC, AU JSK, HAO Q, CHEUNG KH, WU W, LI GR, YUE J: Mechanistic study of TRPM2-Ca(2+)-CAMK2-BECN1 signaling in oxidative stressinduced autophagy inhibition. Autophagy 12: 1340-1354, 2016.

WHO: Health effects of particulate matter. Policy implications for countries in Eastern Europe, Caucasus and central Asia. WHO Regional Office for Europe, Copenhagen, 2013. 
YURUKER V, NAZIROGLU M, SENOL N: Reduction in traumatic brain injury-induced oxidative stress, apoptosis, and calcium entry in rat hippocampus by melatonin: Possible involvement of TRPM2 channels. Metab Brain Dis 30: 223-231, 2015.

ZHANG Q, QIU M, LAI K, ZHONG N: Cough and environmental air pollution in China. Pulm Pharmacol Ther 35: 132-136, 2015.

ZHANG Y, LI XR, CRAILLER JJ, WANG N, WANG MM, YAO JF, ZHONG R, GAO GF, WARD PA, TAN DX, LI XD: Melatonin alleviates acute lung injury through inhibiting the NLRP3 inflammasome. J Pineal Res 60 : 405-414, 2016. 